Research Paper

\title{
The Anti-inflammatory Role of Endometase/Matrilysin-2 in Human Prostate Cancer Cells
}

\author{
Zahraa I. Khamis ${ }^{1}$, Diego A.R. Zorio ${ }^{\boxplus}$, Leland W.K. Chung 3 , and Qing-Xiang Amy Sang ${ }^{1,2, \varpi}$ \\ 1. Department of Chemistry and Biochemistry, Florida State University, Tallahassee, FL 32306, USA; \\ 2. Institute of Molecular Biophysics, Florida State University, Tallahassee, FL 32306, USA; \\ 3. Department of Medicine, The Samuel Oschin Comprehensive Cancer Institute, Cedars-Sinai Medical Center, 8750 Beverly Blvd. Atrium \\ 103, Los Angeles, CA 90048, USA.
}

\begin{abstract}
$\triangle$ Corresponding author: Prof. Qing-Xiang (Amy) Sang, Ph.D., Department of Chemistry and Biochemistry and Institute of Molecular Biophysics, Florida State University, 102 Varsity Way, Tallahassee, FL 32306-4390, USA; Tel: +1-850-644-8683; Fax: +1-850-644-8281; E-mail: qxsang@chem.fsu.edu or Dr. Diego Zorio, Department of Chemistry and Biochemistry, 95 Chieftan Way, Florida State University, Tallahassee, FL 32306-4390, USA; Tel: +1-850-644-8267; Fax: +1-850-644-8281; Email: zorio@chem.fsu.edu.
\end{abstract}

(C) Ivyspring International Publisher. This is an open-access article distributed under the terms of the Creative Commons License (http://creativecommons.org/ licenses/by-nc-nd/3.0/). Reproduction is permitted for personal, noncommercial use, provided that the article is in whole, unmodified, and properly cited.

Received: 2013.01.01; Accepted: 2013.02.07; Published: 2013.03.20

\begin{abstract}
Human endometase/matrilysin-2/matrix metalloproteinase-26 (MMP-26) is an endopeptidase mostly produced by human carcinoma cells. While MMPs are thought to regulate the dynamics of extracellular matrix turnover, new evidence shows that these enzymes may play a critical regulatory role in inflammation. To investigate the role of MMP-26 in inflammation, three different variants of androgen repressed human prostate cancer (ARCaP) cells were investigated in the study: parental, MMP-26 sense cDNA-transfected, and MMP-26 antisense cDNA-transfected ARCaP cells. Protein lysates and RNA from control and genetically modified cells were analyzed by Western blotting and real-time reverse transcription polymerase chain reaction on arrays of genes critical to the inflammatory response. In comparison to parental controls, up-regulation of MMP-26 expression in MMP-26 sense cDNA-transfected cells resulted in a decrease in inflammatory genes expression. Conversely, inflammatory genes were up-regulated in MMP-26 antisense cDNA-transfected cells. Therefore, modulation of MMP-26 levels significantly affects the expression of inflammatory genes, suggesting an anti-inflammatory role of MMP-26. To determine a possible mechanism of action, further analysis, at both transcript and protein levels, revealed a dramatic down-regulation of interleukin-10 receptor B (ILIORB) in MMP-26 antisense cDNA-transfected cells. The low level of ILIORB was inversely correlated with matrix metalloproteinase-9 (MMP-9) expression. Collectively, our data suggest that the deficiency of MMP-26 may promote inflammation via inhibition of ILIORB-mediated signaling. These results propose a novel anti-inflammation function of MMP-26 and could provide novel molecular insight of therapeutic targeting.
\end{abstract}

Key words: matrix metalloproteinase-26, inflammatory genes, real-time reverse transcription polymerase chain reaction, interleukin-10 receptor B, matrix metalloproteinase-9.

\section{Introduction}

Matrix metalloproteinases (MMPs) represent a group of structurally and functionally related zinc-dependent enzymes that are involved in the degradation of nearly all extracellular matrix (ECM) components [1]. In addition, MMPs have been recog- nized to activate other family members and cleave various non-ECM proteins, including growth factors, angiogenic factors, adhesion molecules, cytokines, chemokines, and receptors to modulate different cellular functions [2]. As such, MMPs influence a variety 
of physiological processes like embryo implantation, bone remodeling, cell proliferation, migration, and apoptosis [3, 4]. However, abnormal MMP expression is linked with multiple pathological conditions including cancer, inflammation, and vascular diseases $[5,6]$. The various physiological and pathological processes regulated by MMPs are dependent on their catalytic activity which is tightly controlled at the level of transcription, cellular localization, zymogen activation, and endogenous inhibition by TIMPs (tissue inhibitors of matrix metalloproteinases) [4].

Based on their proteolytic roles, MMPs have been described as modulators of inflammatory processes $[7,8]$. While matrix degradation can help leukocytes migrate to a site of injury, the processing of various inflammatory mediators (i.e., cytokines, chemokines, etc.) represents another route for MMPs to influence inflammation. MMP-mediated proteolysis of cytokines and chemokines alter their bioavailability and activity, which in part affect the inflammatory response and type of leukocytes that are recruited and subsequently activated [7, 8]. For example, cleavage of tumor necrosis factor- $\alpha$ (TNF- $\alpha$ ) by MMP-7 releases an active form capable of regulating macrophage infiltration. Additionally, MMP-9- and MMP-8-mediated processing of CXCL8 generates a more potent chemoattractant [8]. Therefore, MMPs modulate inflammation, but their pro- or anti-inflammatory roles remain questionable. Various studies revealed a major role of MMP-9 in the induction of the immune system while MMP-2 and MMP-3 were shown to provide joint protection in mouse models of rheumatoid arthritis $[9,10]$.

Endometase/matrilysin-2, or MMP-26, is a partially characterized proteinase that was discovered by our group and others [11]. It shares the same structural features of other MMPs, including a signal peptide, a propeptide domain with a unique cysteine switch, and a catalytic domain with the zinc-binding motif. However, MMP-26 lacks the hemopexin-like domain available to other family members [11-14]. In contrast to a majority of MMPs that are associated with the ECM, MMP-26 was found in the intracellular milieu, suggesting unusual roles in cells and tissues $[15,16]$. MMP-26 is expressed in normal, as well as in cancerous cells of epithelial origin such as, endometrium, breast, and prostate [11, 16-18]. Yet, additional studies showed paradoxical results of MMP-26 expression and function. Similar to other tumor-promoting MMPs, MMP-26 expression was found to be significantly higher in human prostate carcinomas when compared to normal and hyperplastic prostate [19]. MMP-26 was also shown to activate pro-MMP-9 and thereby, promote cancer inva- sion [19]. Moreover, $\mathrm{Li}$ et al. show that MMP-26-mediated cleavage of a1-antitrypsin (AAT) correlates with MMP-26 expression in infiltrating leukocytes, suggesting MMP-26 ability to promote inflammation [20]. Unlike other pro-tumorigenic MMPs, the expression of MMP-26 in ductal carcinoma in situ results in favorable prognosis and longer survival of breast cancer patients due to its proteolysis of estrogen receptor $\beta$ (ER $\beta)[21,22]$.

To further understand the role of MMP-26 in inflammation, we studied the expression of genes involved in the inflammatory response within androgen repressed prostate cancer (ARCaP) cell lines. The transfection of MMP-26 sense or antisense cDNA resulted in a dramatic up- or down-regulation of the protein level, respectively. A marked reduction in the invasiveness of antisense transfected cells was also observed with no major difference in the invasive ability of parental and sense transfected cells [19]. The three variants investigated in this study are: (i) parental ARCaP cells; (ii) MMP-26 sense cDNA-transfected ARCaP cells; and (iii) MMP-26 antisense cDNA-transfected ARCaP cells. Our data suggest a previously unrecognized role of MMP-26 deficiency in promoting inflammation by downregulating interleukin-10 receptor B and upregulating MMP-9.

\section{Materials and methods}

\section{Cell culture and transfections}

ARCaP cells were transfected with sense and antisense MMP-26 cDNA as previously described by Zhao et al. [19]. All cells were grown in low-glucose Dulbecco's modified Eagle's medium supplemented with $10 \%$ fetal bovine serum in a humidified chamber containing $5 \% \mathrm{CO}_{2}$ at $37^{\circ} \mathrm{C}$.

\section{RNA extraction and DNase treatment}

RNA was extracted using RNA-Bee ${ }^{\mathrm{TM}}$ isolation reagent (Tel-Test, Inc.) followed by an extra phenol-chloroform extraction and ammonium acetate precipitation. RNA samples were DNase treated using turbo DNA-free ${ }^{\mathrm{TM}}$ following manufacturer's instructions (Life Technologies ${ }^{\mathrm{TM}}$ ). DNA-free RNAs were suspended in nuclease free water. RNA samples were assessed for quality and integrity using the Agilent 2100 Bioanalyzer (Agilent Technologies, Inc). All RNA samples exhibited a high quality RNA, as indicated by the RNA Integrity Number (RIN), and were utilized as templates for first strand cDNA synthesis.

\section{Real time PCR}

RT2ProfilerTM PCR arrays of Human Inflamma- 
tory Cytokines and Receptors (PAHS-011C) were purchased from SuperArray Biosciences and cDNA was synthesized according to manufacturer instructions. Real time PCR was performed using ABI7500 Fast System (Life Technologies ${ }^{\mathrm{TM}}$ ) with SYBR Green dye mix of 96-well $\mathrm{RT}^{2}$ profiler PCR array containing 84 key genes involved in the inflammatory pathway. The PCR reaction was run as follows: one 10 minute cycle at $95^{\circ} \mathrm{C}$ followed by 40 cycles of 15 seconds at $95^{\circ} \mathrm{C}$ and 1 minute at $60^{\circ} \mathrm{C}$. Threshold cycle $\left(C_{t}\right)$ data generated from the real time instrument were analyzed using an excel-based PCR array data analysis template offered by SuperArray Biosciences. All data were normalized to the parental ARCaP control.

\section{Western blot}

Western blots were performed by lysing the cells with lysis buffer (30 mM Tris, $7 \mathrm{M}$ urea, $4 \%$ CHAPS, and protease inhibitor) followed by vortexing for 1 hour and centrifugation for 15 minutes at $15000 \mathrm{~g}$. Protein extraction and quantification was done using a microplate bicinchoninic acid (BCA) protein assay kit as described previously [23]. Aliquots of $25 \mu \mathrm{g}$ of cell lysate were treated with SDS sample buffer and loaded onto a $10 \%$ polyacrylamide gel. The gel was electrophoresed at $50 \mathrm{~V}$ for $30 \mathrm{~min}$ then at $100 \mathrm{~V}$ till the end of the separation. Proteins were subsequently electroblotted onto nitrocellulose membrane at $50 \mathrm{~V}$ for 2 hours at room temperature with membranes then were blocked with 5\% BSA in TBST buffer. Western blot analyses were accomplished using 1 $\mu \mathrm{g} / \mathrm{mL}$ dilution of MMP-26 primary antibody targeting the pro-domain [24], $2 \mu \mathrm{g} / \mathrm{mL}$ dilution of MMP-9 antibody (Oncogene Research Products cat. IM09L), and 1:500 dilution of IL10RB antibody (Santa Cruz Biotechnology Inc cat. sc-69574) all in 1\% BSA. This was followed by incubation with horseradish peroxidase-conjugated secondary antibody against the appropriate species. Band development upon Kodak Scientific Imaging Film (Kodak cat.1651496) was accomplished by addition of a 1:1 ratio of Super Signal West Pico-Stable Peroxidase Solution and Luminol/Enhancer Solution (Pierce, Rockford,IL) and by using Fixer and Replenisher/Developer and Replenisher (Kodak cat.1901859) according to manufacturer's instructions.

\section{Results}

\section{MMP-26 expression in ARCaP cell lines}

To understand the possible functional role of MMP-26 in prostate cancer cells, we took a genetic approach by either overexpressing or knockdown of MMP-26 in ARCaP cells. ARCaP cells were chosen due to their intrinsic property of metastasizing to bone, a known lethal phenotype of human prostate cancer, and high expression of MMP-26 and MMP-9. ARCaP cells have been genetically manipulated to study the potential influence of MMP-26 on prostate cancer progression as we previously reported [19]. $\mathrm{ARCaP}$ cells were transfected with either sense or antisense MMP-26 cDNA, and multiple clones were selected in the presence of G-418 for stable transfectants. MMP-26 protein levels were evaluated in these ARCaP cell lines. Western blot analysis shows MMP-26 protein was significantly increased in AR$\mathrm{CaP}$ cells transfected with sense MMP-26 and a marked down-regulation in antisense transfected cells (Figure 1).

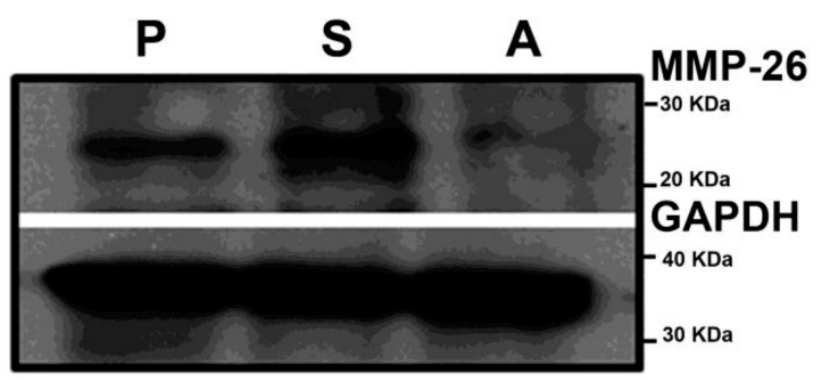

Figure I. Western Blot of MMP-26 in parental ARCaP (P), MMP-26 sense cDNA-transfected ARCaP (S), and MMP-26 antisense cDNA-transfected ARCaP (A) cell lines. An up-regulation of MMP-26 protein exists in sense-transfected cells versus a down-regulation of the protein level in the antisense-transfected cell line. GAPDH was used as loading control.

\section{MMP-26 regulates the expression of inflam- matory genes}

To seek a possible link between MMP-26 and inflammation, we evaluated the levels of inflammatory-related genes expressed in ARCaP cells either with overexpressed or downregulated MMP-26 expression using PCR arrays of human inflammatory cytokines and receptors. RT-PCR data were subjected to analyses by Ingenuity's Pathway Analysis (IPA) software. Transfection of MMP-26 sense or antisense cDNA into ARCaP cells regulated the expression levels of a panel of inflammatory genes (Table 1). Down-regulation of MMP-26 in antisense-transfected cells resulted in significant over-expression of genes involved in the inflammatory response, including CCL4, CXCL3, CCL7, CCL3, and CCL16 (Table 1 \& Figure 2). Accordingly, MMP-26 over-expression in sense-transfected ARCaP cells caused a dramatic down-regulation of the inflammatory genes expression (CCL15, CCL23, 
CXCL5, CXCL2, and CXCL1). Although few genes were regulated in same direction in sense and antisense transfected cells; overall, the data presented here suggests an anti-inflammatory role of MMP-26 metalloprotease.

\section{Effect of MMP-26 modulation on expression of MMP-9 and ILIORB}

The Knock down of MMP-26 exhibited the greatest effect on interleukin 10 receptor B (IL10RB), down-regulating its mRNA level by more than 4000-fold (Table 1). Since IL10RB has known functions in anti-inflammatory response, we examined the levels of its protein expression in these cells. Western blot analysis showed a decrease in IL10RB protein in antisense-transfected ARCaP cells, corroborating real time RT-PCR results (Figure 3, Right). Attempting to link MMP-26 and IL10RB, we investigated the possible pathways, and correlations involving these two genes, using pathway analysis software from Ingenuity Systems, Inc. Surprisingly, MMP-9 was found to be involved in bridging the interaction between MMP-26 and IL10RB (Figure 4). To determine the role of MMP-9 in this crosstalk, Western blot analysis was performed and indicated an inverse expression pattern of MMP-9 to that of IL10RB (Figure 3, Left). Catalytic MMP-9 (the $35 \mathrm{kDa}$ species) was highly expressed in antisense-transfected cells relative to those transfected with sense MMP-26 cDNA. This suggests a potential relationship between MMP-9, MMP-26, and IL10RB.

Table I. Fold change of most affected inflammation-associated genes in MMP-26 sense cDNA- and antisense cDNA-transfected ARCaP cells.

\begin{tabular}{|c|c|c|c|}
\hline Symbol & Description & Sense fold & Antisense fold \\
\hline IL10RB & Interleukin 10 receptor $B$ & 1.0323 & -4686.6287 \\
\hline CCL23 & Chemokine (C-C motif) ligand 23 & -16.1796 & -14.6725 \\
\hline C4A & Complement component 4A (Rodgers blood group) & -69.1807 & -14.3066 \\
\hline CXCL14 & Chemokine (C-X-C motif) ligand 14 & 1.2399 & -12.5151 \\
\hline IL8 & Interleukin-8 & 1.9003 & 12.1993 \\
\hline CCL11 & Chemokine (C-C motif) ligand 11 & -1.1621 & 13.1494 \\
\hline CCL2 & Chemokine (C-C motif) ligand 2 & -3.8312 & 13.6084 \\
\hline CXCL1 & Chemokine (C-X-C motif) ligand 1 (melanoma growth stimulating activity, alpha) & -15.6675 & 15.1047 \\
\hline CXCL2 & Chemokine (C-X-C motif) ligand 2 & -10.6699 & 25.1024 \\
\hline CCL17 & Chemokine (C-C motif) ligand 17 & -3.0077 & 29.3469 \\
\hline CCL4 & Chemokine (C-C motif) ligand 4 & -1.84 & 29.9093 \\
\hline CXCL3 & Chemokine (C-X-C motif) ligand 3 & -6.5266 & 33.932 \\
\hline CCL7 & Chemokine (C-C motif) ligand 7 & 5.6964 & 52.7151 \\
\hline CCL3 & Chemokine (C-C motif) ligand 3 & -1.7995 & 103.4794 \\
\hline CCL16 & Chemokine (C-C motif) ligand 16 & 1.0853 & 372.5222 \\
\hline CXCL5 & Chemokine (C-X-C motif) ligand 5 & -65.8087 & 3.7762 \\
\hline IL1R1 & Interleukin 1 receptor, type I & -29.508 & -3.9604 \\
\hline LTB & Lymphotoxin beta (TNF superfamily, member 3) & -24.8252 & -6.8871 \\
\hline CCL15 & Chemokine (C-C motif) ligand 15 & -23.1171 & 1.7974 \\
\hline IL1F6 & Interleukin 1 family, member 6 (epsilon) & -13.0029 & 1.5134 \\
\hline CXCL6 & Chemokine (C-X-C motif) ligand 6 (granulocyte chemotactic protein 2) & -12.5348 & 8.3752 \\
\hline CCL21 & Chemokine (C-C motif) ligand 21 & -12.5088 & 1.508 \\
\hline CCR2 & Chemokine (C-C motif) receptor 2 & -4.8029 & -1.0072 \\
\hline CCR4 & Chemokine (C-C motif) receptor 4 & -2.2115 & -1.4217 \\
\hline CCR5 & Chemokine (C-C motif) receptor 5 & -3.5227 & 3.1016 \\
\hline IL9R & Interleukin 9 receptor & -6.4991 & 2.72 \\
\hline
\end{tabular}




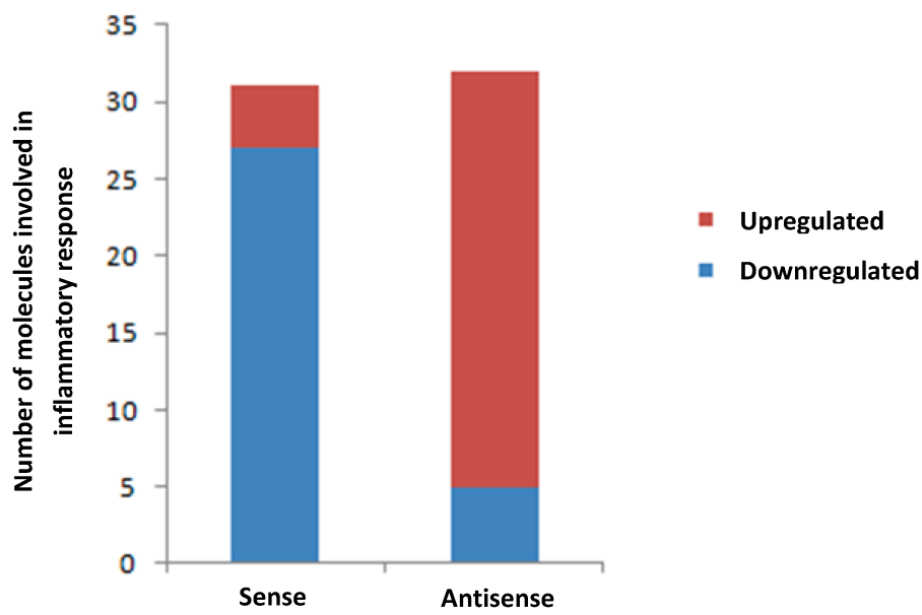

Figure 2. Bar diagram showing alteration of genes involved in inflammatory response in MMP-26 sense cDNA- and MMP-26 antisense cDNA-transfected ARCaP cells normalized to the parental ARCaP control. Red shows the number of genes up-regulated and blue indicates the number of down-regulated genes.

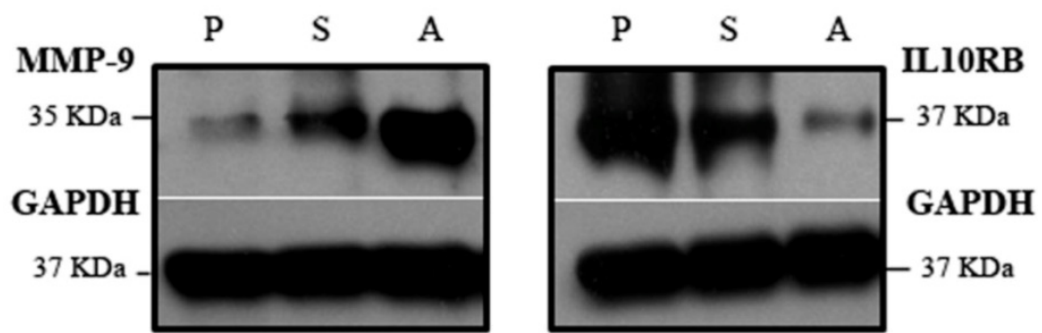

Figure 3. Western blot of MMP-9 and ILIORB in parental ARCaP (P), MMP-26 antisense cDNA- (A), and MMP-26 sense cDNA-transfected (S) cell lines. A major up-regulation of MMP-9 expression and dramatic down-regulation of ILIORB occurred in the antisense-transfected cell line. GAPDH was used as loading control.

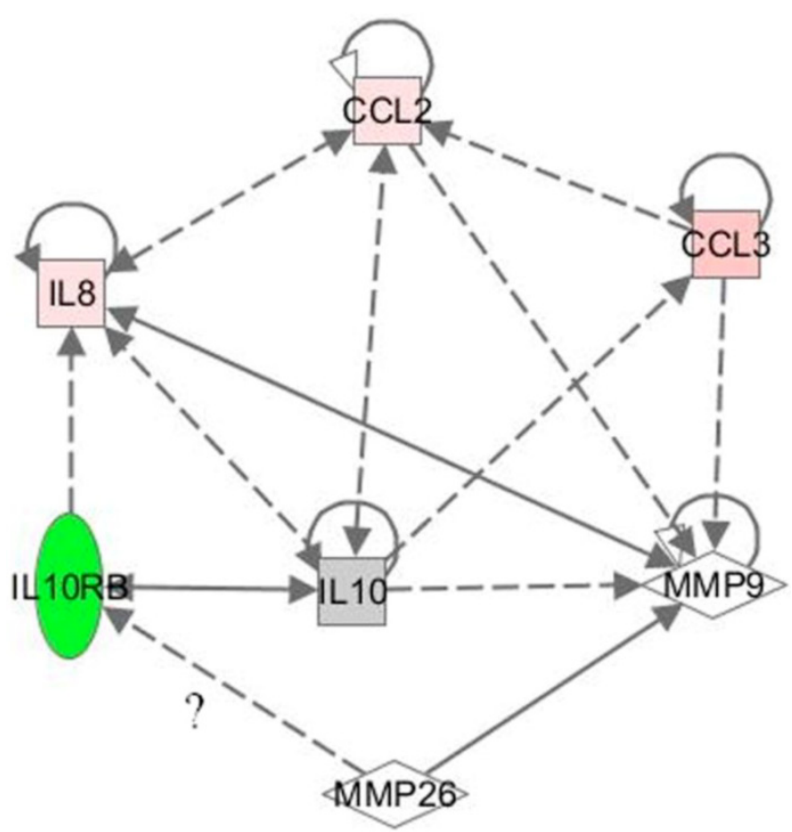

Figure 4. Network showing the correlation between MMP-26, MMP-9, ILIORB, CCL2, IL8, and CCL3. Dotted lines reflect indirect relationships while bold line indicates a direct one. MMP-26/ILIORB relationship is questionable. The shapes represent the following; square: cytokine, rhombus: peptidase, and oval: transmembrane receptor. Green color reflects a down-regulation, red color an up-regulation, and gray color no change. The color represents the change in the MMP-26 antisense cDNA-transfected ARCaP cells. The more intense the color, the stronger is the modulation. 


\section{Discussion}

MMPs are considered important regulators of inflammatory disease [8]. In addition to leukocyte trafficking, MMPs can modulate inflammation by direct processing of a myriad of inflammatory mediators, including chemokines and cytokines. This proteolysis might inactivate, augment, or antagonize their functions, thereby affecting the outcome of an inflammatory response [8, 25]. Although targeted inhibition of MMPs was used as a novel therapeutic option to treat some inflammatory conditions [5], the exact roles of these enzymes in inflammation are still underway.

MMP-26 is an under-explored matrix metalloproteinase that has not been well characterized in inflammation. ARCaP cells are highly invasive and metastatic and express both MMP-26 and MMP-9, making them an appropriate model to study MMP-26 function [19]. In an attempt to elucidate this function, we investigated the effect of MMP-26 on the expression of inflammatory genes. Over-expression of MMP-26 in ARCaP cells decreased the expression of inflammatory genes, while its down-regulation exhibited an opposite effect (Figure 2). This suggests a novel anti-inflammatory role for MMP-26.

Among the expressed inflammatory genes, IL10RB is significantly down-regulated upon MMP-26 knockdown (Figure 3). IL10RB, originally known as orphan interferon receptor family member CRFB4/CRF2-4, is the accessory subunit of the IL10 receptor complex (IL10R) [26]. For IL10 to transduce signals into a cell, the ligand-binding chain of the IL-10R, IL10RA, is required [26, 27]. IL10 signaling is an anti-inflammatory pathway that, through IL10R, inhibits cytokine production by $\mathrm{T}$ cells and natural killer (NK) cells, and suppresses antigen presenting cells (APC) cytokine expression [28]. Sequential assembly of IL10 with IL10RA and IL10RB engages the Janus tyrosine kinases Jak1 and Tyk2, which are associated with IL10RA and IL10RB respectively. IL10RB is constitutively expressed on a wide variety of cells and tissues whereas IL10RA expression is more limited [27]. Unlike IL10RA, IL10RB is a common component of the functional receptors for several additional cytokines (IL22, IL26, and the IFN- $\lambda$ proteins) [26]. Produced by leukocytes, cytokines requiring IL10RB, with the exception of IL10, exert their actions on non-hematopoietic cells and therefore, has no functional effect on leukocytes [26]. Surprisingly, mice deficient in IL10RB, similar to IL10 knockout mice [29], were shown to develop severe enterocolitis, suggesting a pivotal role of IL10RB in inflammatory diseases [30]. Based on this, we hypothesize that
MMP-26 knockdown induces inflammation by suppressing IL10RB expression through a yet unknown mechanism.

Examining the crosstalk between MMP-26 and IL10RB, Ingenuity's Pathway Analysis software was used and showed a relationship between MMP-9, MMP-26, and IL10RB (Figure 4). IL10 signaling pathway is known to decrease the expression and activity of MMP-9 [27, 31]. However, MMP-9 expression is induced by tumor necrosis factor (TNF) cytokine in mouse colon epithelial cells lacking TNF receptor 2 (TNFR2 $-/-$ ), but not TNFR1 $\%$, suggesting that TNFR1 is required for this effect [32]. TNF receptors are distinct receptors [33], whereas IL10 receptor is heteromeric and thus; requires the presence of IL10RA and IL10RB for signal transduction. Therefore, it is believed that IL10RB deficiency will cause aberrant IL10 signaling, subsequently inducing MMP-9 expression. In addition, some cytokines such as CCL3, CCL2, and IL8 (Table 1), which are overexpressed in the antisense-transfected ARCaP cells, are capable of inducing MMP-9 expression [34, 35]. Collectively, we suspected that cells deficient in MMP-26 may induce inflammation via inhibition of IL10RB and up-regulation of MMP-9. To address this issue, Western blot analysis was performed and showed a significant increase in MMP-9 accompanied by down-regulation of IL10RB in the antisense-transfected cell line (Figure 3), supporting our hypothesis. This up-regulation of MMP-9 is limited to its $35 \mathrm{kDa}$ active species, which is reported to possess highly efficient proteolytic activity for gelatins, collagen type IV, and fibronectin and produced by autocatalytic processing of the $82 \mathrm{kDa}$ pro- form [36]. Since MMP-9 is up-regulated in the sense-transfected cell line, and further increased in antisense-transfected cells, its expression is not believed to be correlated or dependent upon MMP-26, even though MMP-26 is known to cleave pro-MMP-9 [19]. Specifically, knockdown of MMP-26, through the modulation of the expression of genes such as IL10RB, may affect MMP-9 expression. Moreover, the inverse pattern of expression of IL10RB and MMP-9 in sense- and antisense-transfected cells suggests an inverse correlation between these two proteins (Figure 3).

Recently, MMPs have been implicated in regulating gene expression. Genome wide studies of MT1-MMP in cancer have led to the identification of target genes that are functionally associated with MT1-MMP [37]. Our study reveals, for the first time, the effect of MMP-26 modulations in cancer cells on the expression of some genes, in particular inflammatory genes. The identification of some MMP-26 target genes is an important step in understanding the 
molecular mechanisms that govern this MMP in tumorigenesis. Additional extensive studies are currently in progress to identify more MMP-26 target genes and to elucidate its mechanism of action in changing gene expression.

MMP-26 deficiency caused an increase in the expression of inflammatory genes through down-regulation of IL10RB and subsequent up-regulation of MMP-9. It is plausible that this induction of MMP-9 expression is not only dependent on modulation of IL10RB, but also on the alteration of the expression of other genes that provoke or reduce MMP-9 expression. Over-expressing MMP-26 in AR$\mathrm{CaP}$ cells resulted in lower levels of inflammatory genes indicating a potential role of MMP-26 in inflammation. MMP-26 mechanisms of action in inflammation within sense- and antisense-transfected cells may involve different pathways and warrant further investigation.

\section{Conflict of Interest}

The authors declare no conflict of interest.

\section{Acknowledgments}

This work was supported in part by grants DAMD17-02-1-0238 and W81XWH-07-1-0225 from DOD, US Congressionally Directed Medical Research Programs, and grants from Florida State University (to Q.-X.S.); and Florida State University Research Foundation (to D.A.R.Z).

\section{References}

1. Egeblad M, Werb Z. New functions for the matrix metalloproteinases in cancer progression. Nature Reviews Cancer. 2002; 2: 161-174.

2. Noel A, Jost M, Maquoi E. Matrix metalloproteinases at cancer tumor-host interface. Seminars in Cell \& Developmental Biology. 2008; 19: 52-60.

3. Rowe RG, Weiss SJ. Breaching the basement membrane: Who, when and how? Trends in Cell Biology. 2008; 18: 560-574.

4. Page-McCaw A, Ewald AJ, Werb Z. Matrix metalloproteinases and the regulation of tissue remodelling. Nature Reviews Molecular Cell Biology. 2007; 8: 221-233.

5. Hu JL, Van den Steen PE, Sang QXA, et al. Matrix metalloproteinase inhibitors as therapy for inflammatory and vascular diseases. Nature Reviews Drug Discovery. 2007; 6: 480-498.

6. Lopez-Otin C, Matrisian LM. Tumour micro environment - opinion emerging roles of proteases in tumour suppression. Nature Reviews Cancer. 2007; 7: 800-808.

7. Parks WC, Wilson CL, Lopez-Boado YS. Matrix metalloproteinases as modulators of inflammation and innate immunity. Nature Reviews Immunology. 2004; 4: 617-629.

8. Manicone AM, McGuire JK. Matrix metalloproteinases as modulators of inflammation. Seminars in Cell \& Developmental Biology. 2008; 19: 34-41.

9. Itoh $\mathrm{T}$, Matsuda $\mathrm{H}$, Tanioka $\mathrm{M}$, et al. The role of matrix metalloproteinase- 2 and matrix metalloproteinase- 9 in antibody-induced arthritis. Journal of Immunology. 2002; 169: 2643-2647.

10. Mudgett JS, Hutchinson NI, Chartrain NA, et al. Susceptibility of stromelysin 1-deficient mice to collagen-induced arthritis and cartilage destruction. Arthritis and Rheumatism. 1998; 41: 110-121.

11. Park HI, Ni J, Gerkema FE, et al. Identification and characterization of human endometase (matrix metalloproteinase-26) from endometrial tumor. Journal of Biological Chemistry. 2000; 275: 20540-20544.
12. Uria JA, Lopez-Otin C. Matrilysin-2, a new matrix metalloproteinase expressed in human tumors and showing the minimal domain organization required for secretion, latency, and activity. Cancer Research. 2000; 60: 4745-4751.

13. Marchenko GN, Ratnikov BI, Rozanov DV, et al. Characterization of matrix metalloproteinase-26, a novel metalloproteinase widely expressed in cancer cells of epithelial origin. Biochemical Journal. 2001;356: 705-718.

14. de Coignac AB, Elson G, Delneste $Y$, et al. Cloning of mmp-26 - a novel matrilysin-like proteinase. European Journal of Biochemistry. 2000; 267: 3323-3329.

15. Park HI, Turk BE, Gerkema FE, et al. Peptide substrate specificities and protein cleavage sites of human endometase/matrilysin-2/matrix metalloproteinase-26. Journal of Biological Chemistry. 2002; 277: 35168-35175.

16. Marchenko ND, Marchenko GN, Weinreb RN, et al. Beta-catenin regulates the gene of mmp-26, a novel matrix metalloproteinase expressed both in carcinomas and normal epithelial cells. International Journal of Biochemistry \& Cell Biology. 2004; 36: 942-956.

17. Zhao YG, Xiao AZ, Park HI, et al. Endometase/matrilysin-2 in human breast ductal carcinoma in situ and its inhibition by tissue inhibitors of metalloproteinases-2 and -4: A putative role in the initiation of breast cancer invasion. Cancer Research. 2004; 64: 590-598.

18. Tunuguntla R, Ripley D, Sang QXA, et al. Expression of matrix metalloproteinase-26 and tissue inhibitors of metalloproteinases timp-3 and-4 in benign endometrium and endometrial cancer. Gynecologic Oncology. 2003; 89: 453-459.

19. Zhao YG, Xiao AZ, Newcomer RG, et al. Activation of pro-gelatinase $b$ by endometase/matrilysin-2 promotes invasion of human prostate cancer cells. Journal of Biological Chemistry. 2003; 278: 15056-15064.

20. Li W, Savinov AY, Rozanov DV, et al. Matrix metalloproteinase-26 is associated with estrogen-dependent malignancies and targets alpha 1-antitrypsin serpin. Cancer Research. 2004; 64: 8657-8665.

21. Savinov AY, Remacle SG, Golubkov RS, et al. Matrix metalloproteinase 26 proteolysis of the nh2-terminal domain of the estrogen receptor beta correlates with the survival of breast cancer patients. Cancer Research. 2006; 66: 2716-2724.

22. Harris HA, Albert LM, Leathurby $\mathrm{Y}$, et al. Evaluation of an estrogen receptor-beta agonist in animal models of human disease. Endocrinology. 2003; 144: 4241-4249.

23. Khamis ZI, Iczkowski KA, Sahab ZJ, et al. Protein profiling of isolated leukocytes, myofibroblasts, epithelial, basal, and endothelial cells from normal, hyperplastic, cancerous, and inflammatory human prostate tissues. Journal of Cancer. 2010; 1: 70-79.

24. Lee S, Desai KK, Iczkowski KA, et al. Coordinated peak expression of mmp-26 and timp-4 in preinvasive human prostate tumor. Cell Research. 2006; 16: 750-758.

25. Van Lint $\mathrm{P}$, Libert $\mathrm{C}$. Chemokine and cytokine processing by matrix metalloproteinases and its effect on leukocyte migration and inflammation. Journal of Leukocyte Biology. 2007; 82: 1375-1381.

26. Donnelly RP, Sheikh F, Kotenko SV, et al. The expanded family of class ii cytokines that share the il-10 receptor-2 (il-10r2) chain. Journal of Leukocyte Biology. 2004; 76: 314-321.

27. Moore KW, Malefyt RD, Coffman RL, et al. Interleukin-10 and the interleukin-10 receptor. Annual Review of Immunology. 2001; 19: 683-765.

28. Asadullah K, Sterry W, Volk HD. Interleukin-10 therapy - review of a new approach. Pharmacological Reviews. 2003; 55: 241-269.

29. Kuhn R, Lohler J, Rennick D, et al. Interleukin-10-deficient mice develop chronic enterocolitis. Cell. 1993; 75: 263-274.

30. Spencer SD, Di Marco F, Hooley J, et al. The orphan receptor crf2-4 is an essential subunit of the interleukin 10 receptor. Journal of Experimental Medicine. 1998; 187: 571-578.

31. Lacraz S, Nicod LP, Chicheportiche R, et al. Il-10 inhibits metalloproteinase and stimulates timp-1 production in human mononuclear phagocytes. Journal of Clinical Investigation. 1995; 96: 2304-2310.

32. Jones DS, Yan F, Wilson G, et al. Tumor necrosis factor (tnf) induces matrix metalloproteinase-9 (mmp-9) expression and activity in mouse colon. Gastroenterology. 2004; 126: A92-a93.

33. Thommesen L, Laegreid A. Distinct differences between tnf receptor 1-and tnf receptor 2-mediated activation of nf kappa b. Journal of Biochemistry and Molecular Biology. 2005; 38: 281-289.

34. Chan A, Hummel V, Weilbach FX, et al. Phagocytosis of apoptotic inflammatory cells by microglia downregulates chemotactic function and attraction of encephalitogenic t-cells. Glia. 2003; :40-40.

35. Opdenakker G, Van den Steen PE, Van Damme J. Gelatinase b: A tuner and amplifier of immune functions. Trends in Immunology. 2001; 22: 571-579. 
36. Ries C, Pitsch T, Mentele R, et al. Identification of a novel $82 \mathrm{kda}$ prommp-9 species associated with the surface of leukaemic cells: (auto-)catalytic activation and resistance to inhibition by timp-1. Biochemical Journal. 2007; 405: 547-558.

37. Rozanov DV, Savinov AY, Williams R, et al. Molecular signature of mt1-mmp: Transactivation of the downstream universal gene network in cancer. Cancer Research. 2008; 68: 4086-4096. 